\title{
An integrated OPF dispatching model with wind power and demand response for day-ahead markets
}

\author{
Ricardo Moreno, Johan Obando, Gabriel Gonzalez \\ Energy and Mechanical Department, Universidad Autónoma de Occidente, Cali, Colombia
}

\begin{abstract}
Article Info
Article history:

Received Aug 22, 2018

Revised Mar 12, 2019

Accepted Apr 3, 2019

\section{Keywords:}

Demand response

Electricity markets

Monte-Carlo simulations

Optimal power flow (OPF)

Wind power

ABSTRACT

In the day-ahead dispatching of network-constrained electricity markets, renewable energy and distributed resources are dispatched together with conventional generation. The uncertainty and volatility associated to renewable resources represents a new paradigm to be faced for power system operation. Moreover, in various electricity markets there are mechanisms to allow the demand participation through demand response (DR) strategies. Under operational and economic restrictions, the operator each day, or even in intra-day markets, dispatchs an optimal power flow to find a feasible state of operation. The operation decisions in power markets use an optimal power flow considering unit commitment to dispatch economically generation and DR resources under security restrictions. This paper constructs a model to include demand response in the optimal power flow under wind power uncertainty. The model is formulated as a mixed-integer linear quadratic problem and evaluated through Monte-Carlo simulations. A large number of scenarios around a trajectory bid captures the uncertainty in wind power forecasting. The proposed integrated OPF model is tested on the standard IEEE 39-bus system.
\end{abstract}

Copyright ( 12019 Institute of Advanced Engineering and Science. All rights reserved.

\section{Corresponding Author:}

Ricardo Moreno,

Energy and Mechanical Department,

Universidad Autónoma de Occidente,

Cali, Colombia.

Email: rmoreno@uao.edu.co

\section{INTRODUCTION}

Nowadays, the power system operation involves the competitive participation of renewable generation and DR resources. Wind power generators are participating in electricity markets under different incentives and the power system operator faces an important challenge related with the wind power intermittency and unpredictability [1-3]. In this context, the integration of demand response resources represents a new resource to be allocated in the power system operation [4]. The integration of DR resources in the markets leads to technical and economic challenges associated with the integration of DR resources into the reserve markets. The focus of this paper is on the formulation of an integrated model to dispatch economically a power system under wind power integration and DR participation.

This paper provides a day-ahead dispatching framework including the integration of wind power generation and demand response resources in the OPF formulation. Specifically, the model proposed in this paper is formulated as a mixed-integer linear quadratic optimization problem, and the evaluation is performed through Monte-Carlo simulations to capture the uncertainty related with the wind power generation. DR resources are modeled as dispatchable loads, which indicates the demand tendency to shed its consumption at determined price.

The wind power output has an intermittent nature, so, the integration of wind power generation requires ancillary services such as regulation, contingency reserve and others to compensate wind power 
ramps. The generation system reliability of the system may be reduced in case of unpredicted decreases in wind power because the available ramping capability of the system may not be sufficient to accommodate those changes. The day-ahead dispatching with wind power has been addressed widely. The optimal power flow (OPF) formulation have been extended to account for the variable nature of wind power generation. For instance, in [5-8], the intermittent nature of wind power generation is captured using probabilistic techniques. A stochastic unit commitment model is presented in [9], where authors propose a framework to quantify the impact of large-scale wind power integration into power systems. The uncertainty in the wind generation is addressed by means of scenarios and demands are considered to be fixed. Others authors have suggested stochastic optimization (SO) based on scenarios to cope with wind power uncertainty in the unit commitment problem [10-12]. SO employs several scenarios along with their associated probabilities to simulate possible uncertainties during the period. In [13], an optimal generation scheduling method including renewable energy, distributed resorues and storage systems is solved using a particle swan optimization algorithm. In addition, in [14], the auhors propose an enhanced genetic algorithm to solve the optimal power flow.

On the other hand, the integration of demand side resources into electricity markets has drawn a lot of attention. DR is a strategy to utilize electricity demand as a distributed resource with real possibilities to improve efficiency and reliability of electricity networks. Usually, the demand in power system is considered inelastic to the prices. However, a substantial amount of electricity demand is elastic such as plug-in electric vehicle (PEV) [15] charging batteries, heating ventilation, air conditioning, and this report [16] indicates that one third of residential demand in U.S. is flexible.

Several studies are researching about the participation of demand side resources in the procurement of energy and reserve services. Seminal studies [17-19] have developed pool based market structures considering the participation of demand side resources into the energy and reserve markets. The demand side resources (i.e., DR resources) are technically capable of providing ancillary services given the flexibility and the possibility to alleviate large and unexpected wind ramp events [20, 21]. Distribution companies or aggregators usually manage DR resources [22]. The aggregators represent technically and financially various users in order to bid DR reductions in electricity markets. This paper addresses the day-ahead dispatching including wind power bids and DR bids.

The paper is organized as follows. The problem formulation is presented in Section II. In Section III, the proposed procedure is tested using the IEEE 39-bus test system. The results are analyzed and discussed. Section IV provides some concluding remarks.

\section{PROBLEM FORMULATION}

The notation for the OPF dispatching model including wind power generations and DR resources is expressed in terms of power generation for each unit, the load following reserves and the binary commitment variable for thermal units. The complete set of variables are described as follows:

\subsection{Notation}

$t \quad$ Index over time periods.

$T \quad$ Set of indices of time periods in the planning horizon, typically $\left\{1 \ldots n_{t}\right\}$.

$i \quad$ Index over injections (generation units, dispatchable or curtailable loads).

$j \quad$ Index over scenarios.

$I^{t} \quad$ Indices of all units (generators) available for dispatch in any time $t$.

$f \quad$ Index of wind farms.

$N_{F} \quad$ Set of indices of all units (wind farms and generators) available for dispatch in any time $t$

$b \quad$ Index of loads.

$P_{D_{M A X}} \quad$ Max., power demand for unit $i$ at time $t$.

$N_{B} \quad$ Set of indices of all loads at time $t$.

$F_{t i} \quad$ Load flexibility of demand for unit $i$ at time $t$.

$p^{t i j} \quad$ Active injection for unit $i$ of scenario $j$ at time $t$.

$p_{w}^{t f} \quad$ Wind power forecast as offered in the market for unit $f$ at time $t$

$P_{D}^{t i} \quad$ Real power demand for unit $i$ of scenario $j$ at time $t$.

$C_{P}^{t i}($.$) \quad Cost function for active injection i$ at time $t$.

$C_{D}^{t i}($.$) Cost function of upward and downward regulation of the demand from unit i$ at time $t$.

$D^{t b} \quad$ Demand power at time $t$.

An integrated OPF dispatching model with wind power and demand response for day ... (Ricardo Moreno) 
$\delta_{+}^{t i}, \delta_{\underline{t}}^{t i} \quad$ Upward/downward load-following ramping reserves needed from unit $i$ at time $t$ for transition to time $t+1$.

$C_{\delta+}^{i j}(),. C_{\delta-}^{i n}($.$) Cost of upward and downward load-following ramp. Reserve for unit i$ at time $t$.

$P_{M I N}^{i j}, P_{M A X}^{t i j}$ Limits on active injection for unit $i$ in the scenario $j$ at time $t$.

$\delta_{M A X+}^{i}, \delta_{M A X-}^{i}$ Upward/downward load-following ramping reserve limits for unit $i$.

$u^{t i} \quad$ Binary commitment state for unit $i$ in period $t$.

$v^{t i}, w^{t i}$ Binary startup and shutdown states for unit $i$ in period $t$.

$C_{v}^{t i}, C_{w}^{t i}$ Startup and shutdown costs for unit $i$ at time $t$

$\alpha_{+}^{t i}, \alpha^{t i}$ Startup and shutdown costs for unit $i$ at time $t$

\subsection{Formulation}

The problem formulation is expressed as a mixed-integer linear quadratic optimization problem (MILP), where the optimization variable $x$ is comprised of all the $p, \delta_{+}^{t i}, \delta^{t i}, u, \quad v$ and $w$ variables corresponding to power generation for each unit, the load following reserves and the binary commitment variable for thermal units.

Objective Function: The objective is expressed as the minimization $f(x)$

$$
\min _{x} f(x)
$$

Subject to

$$
\begin{aligned}
& g(x)=0 \\
& h(x) \leq 0 \\
& x_{\min } \leq x \leq x_{\max }
\end{aligned}
$$

where $f(x)$ is comprised of three components.

$$
f(x)=f_{p}(p)+f_{l f}\left(\delta_{+}, \delta_{-}\right)+f_{u c}(u, w)+f_{d r}(u, w)
$$

Cost of active power dispatch

$$
f_{p}(p)=\sum_{t \in T} \sum_{i \in I^{t}}\left[C_{P}^{t i}\left(p^{t i j}\right)\right]
$$

Cost of load-following ramp reserves

$$
f_{l f}\left(\delta_{+}, \delta_{-}\right)=\sum_{t \in T} \sum_{i \in I^{t}}\left[C_{\delta+}^{t i}\left(\delta_{+}^{t i}\right)+C_{\delta_{-}}^{t i}\left(\delta_{-}^{t i}\right)\right]
$$

Startup and shutdown cost

$$
f_{u c}(v, w)=\sum_{t \in T} \sum_{i \in I^{t}}\left[C_{v}^{t i} v^{t i}+C_{w}^{t i} w^{t i}\right]
$$

Cost of demand response

$$
f_{d r}(v, w)=\sum_{t \in T} \sum_{i \in I^{t}}\left[C_{D}^{t i}\left(P_{D}^{t i j}\right)\right]
$$

This minimization is subject to the following constraints, for all: $t \in T$ all $j \in J^{T}$ and all $i \in I^{t}$ : 
Constraints:

Power balance constraints,

$$
\sum_{i \in I^{T}} p^{t i j}+\sum_{i \in I^{T}} P_{D}^{t i j}+\sum_{f \in N_{F}} p_{w}^{t j j}=\sum_{b \in N_{B}} D^{t b}
$$

Nonlinear transmission flow and voltage limits as inequality constraints,

$$
h^{t j}\left(V^{t j}, p^{t j}\right) \leq 0
$$

Load-following ramping limits and reserves,

$$
\begin{aligned}
& 0 \leq \delta_{+}^{t i} \leq \delta_{\max +}^{t i} \\
& 0 \leq \delta^{t i} \leq \delta_{\max -}^{t i}
\end{aligned}
$$

Injection limits and commitments,

$$
u^{t i} P_{\min }^{t i j} \leq p^{t i j} \leq u^{t i} P_{\max }^{t i j}
$$

Startup and shutdown events,

$$
u^{t i}-u^{(t-1) i}=v^{t i}-w^{t i}
$$

Integer constraints,

$$
u^{t i} \in\{0,1\}, \quad v^{t i} \in\{0,1\}, w^{t i} \in\{0,1\}
$$

Flexibility interval of the demand

$$
0 \leq F_{t i} \leq P_{D \max }
$$

\section{SIMULATIONS RESULTS}

The IEEE 39-bus test system is examined in this section to test the integrated model that considers wind power generation DR resources. The day-ahead dispatching framework proposed as a MILP problem is solved using GUROBI 7.5.1 [23] under the Matpower platform [24]. The case IEEE 39-bus test system includes 10 generators; the data is listed in Table 1. The cost data are equal to report in [25] and [26]. Table 2 lists the quadratic cost functions for each generator in the IEEE 39-bus system according to [27]. The system daily load curve is shown in Figure 1 with a maximum peak of $4531 \mathrm{MW}$ at hour 20 and a minimum of 1840 at hour 3.

Table 1. Generator data for the IEEE 39 bus system

\begin{tabular}{ccccccc}
\hline $\begin{array}{c}\text { Gen. } \\
\#\end{array}$ & $C_{\delta+}$ & $C_{\delta-}$ & $C_{v}$ & $C_{w}$ & $P_{\min }$ & $P_{\max }$ \\
\hline 1 & 6.9 & 6.9 & 920 & 736 & 0 & 250 \\
2 & 6.9 & 6.9 & 920 & 736 & 0 & 678 \\
3 & 6.9 & 6.9 & 920 & 736 & 0 & 650 \\
4 & 6.9 & 6.9 & 920 & 736 & 0 & 632 \\
5 & 6.9 & 6.9 & 920 & 736 & 0 & 508 \\
6 & 6.9 & 6.9 & 920 & 736 & 0 & 650 \\
7 & 6.9 & 6.9 & 920 & 736 & 0 & 560 \\
8 & 6.9 & 6.9 & 920 & 736 & 0 & 540 \\
9 & 6.9 & 6.9 & 920 & 736 & 0 & 830 \\
10 & 6.9 & 6.9 & 920 & 736 & 0 & 1000 \\
\hline
\end{tabular}


Table 2. Cost functions

\begin{tabular}{cc}
\hline Gen. & Cost Function $[\$]$ \\
\hline 1 & $C_{1}=0.00194 P^{2}+7.85 P+310$ \\
2 & $C_{2}=0.0035 P^{2}+8.5 P+260$ \\
3 & $C_{3}=0.00482 P^{2}+7 P+78$ \\
4 & $C_{4}=0.00128 P^{2}+6.4 P+459$ \\
5 & $C_{5}=0.0024 P^{2}+6 P+80$ \\
6 & $C_{6}=0.0032 P^{2}+5.8 P+400$ \\
7 & $C_{7}=0.0053 P^{2}+6.24 P+120$ \\
8 & $C_{8}=0.00185 P^{2}+8.4 P+60$ \\
9 & $C_{9}=0.0025 P^{2}+5.75 P+450$ \\
10 & $C_{10}=0.00142 P^{2}+8.2 P+510$ \\
\hline
\end{tabular}

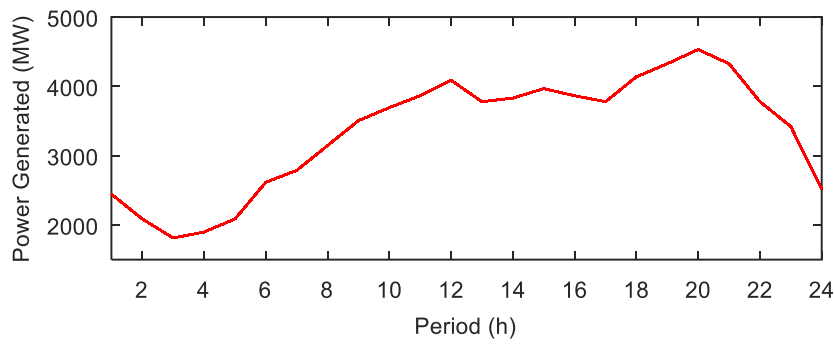

Figure 1. System daily load curve

This model considers a wind power integration level of $20 \%$ with respect to the peak load level. A large number of scenarios around a trajectory bid captures the uncertainty in wind power forecasting by the wind power generator as shown in Figure 2.

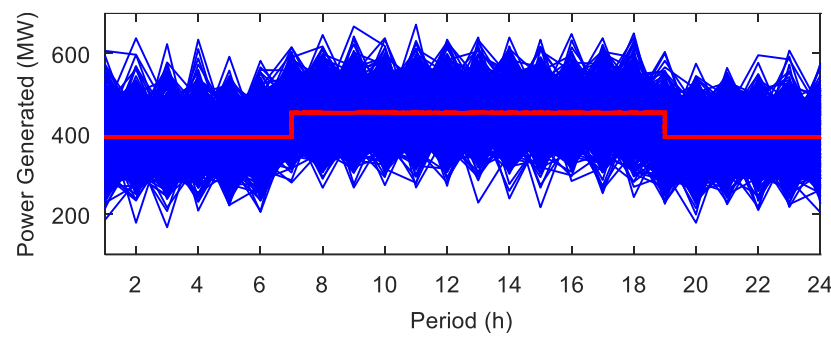

Figure 2. Wind power generation profile

DR offers incentives designed to induce lower electricity use at times of high market price. For this simulation, four loads provide demand response services, and the power quantity, which they are willing to reduce in a certain time, is $1295 \mathrm{MW}$. The incentives represented as cost functions are shown in Table 3.

Table 3. Load data for the demand response

\begin{tabular}{cccc}
\hline Load & Bus & Demand Power $[\mathrm{MW}]$ & Cost Function $[\$]$ \\
\hline 1 & 8 & 522 & $C_{1}=0.00128 P^{2}+6.4 P+459$ \\
2 & 15 & 320 & $C_{2}=0.00128 P^{2}+6.4 P+459$ \\
3 & 23 & 247.5 & $C_{3}=0.00128 P^{2}+6.4 P+459$ \\
4 & 28 & 206 & $C_{4}=0.00128 P^{2}+6.4 P+459$ \\
\hline
\end{tabular}


For five hundred (500) scenarios, this paper quantifies the demand response frequency and he magnitude in MW for each load. Each scenario runs over 24 hours according to the system daily load and it considers a forecasted trajectory for the wind integration. In Figure 3, can observe the frequency and quantity of flexibility for the load in the bus 8. It is obeserved the demand response is maximum all the time. Each time that this load is shed, it is shed at 522 MW. While that for the load in the bus 15, Figure 4 shows sometimes the quantity of DR required is around $270 \mathrm{MW}, 295 \mathrm{MW}$, and $305 \mathrm{MW}$, although the $75 \%$ of time the DR required is at the maximum value. The results for demand response in the bus 23, see Figure 5, are similar to the results in the Figure 4, almost all the time the DR is dispatched at maximum. In Figure 6, the load in the bus 28 exhibit a similar dispatching to the loads in the buses 15 and 23 .

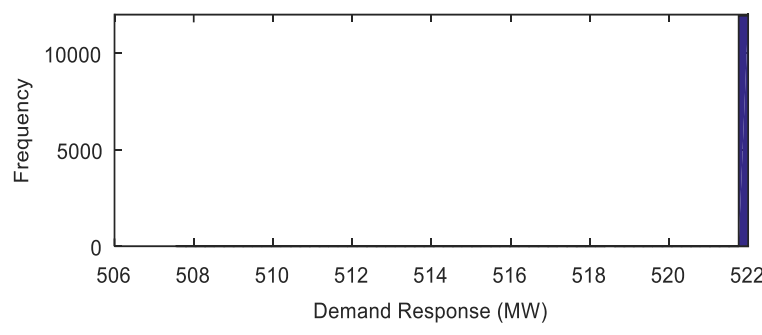

Figure 3. Demand response in the bus 8

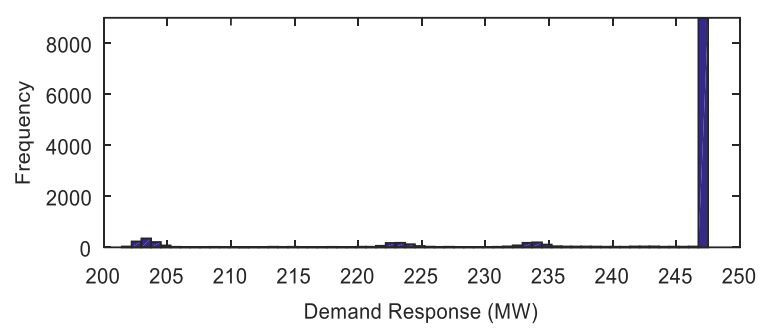

Figure 5. Demand response in the bus 23

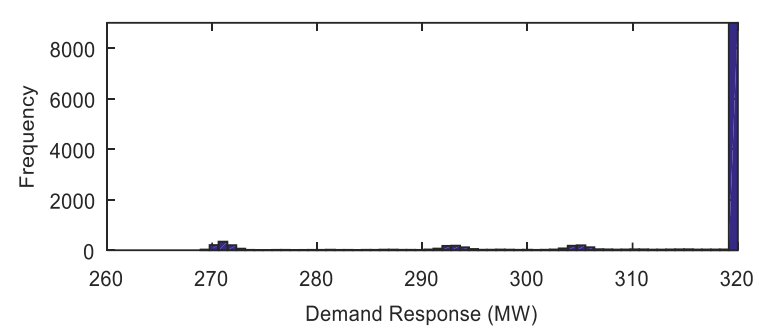

Figure 4. Demand response in the bus 15

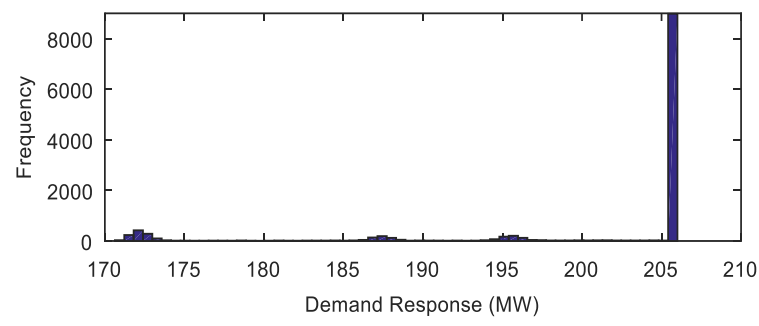

Figure 6. Demand response in the bus 28

Now, in Figure 7 shows the percentage of DR with respect to the available capacity. It is observed that in the hour 3, 13, 16 and 17 for the 24 horizon planning, the DR is not dispatched at maximum. For instance, in the hour 13, the load in the 28 responds at $40 \%$ for the maximum value available. At the peak demand for the system, at hour 20, all loads are dispatched as DR resources.

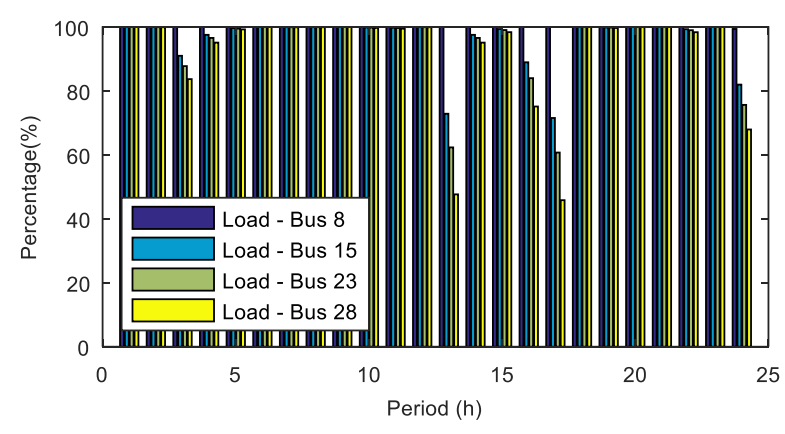

Figure 7. Demand response for all loads in the power system

In order to quantify the benefits for DR participation in the power system operation, the probability density function for the power generation cost is calculated in the 24-hour horizon. The realizations correspond to the trajectories generated around the bid made by the wind power generator. Figure 8 shows the power generation cost under 500 scenarios with DR available for dispatching while the Figure 9 shows the power 
generation cost without DR available. In order to compare the results for both cases, mean and standard deviation for each case are calculated. The savings for the power generation cost is $\$ 8271$. The result represents the savings can be made in the power generation cost if the demand is flexible. Table 4 compares the mean and variance parameters for both cases, with DR and without DR.

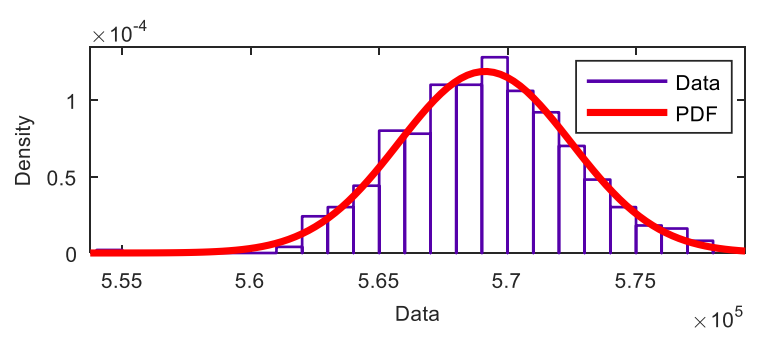

Figure 8. Cost objective function with DR

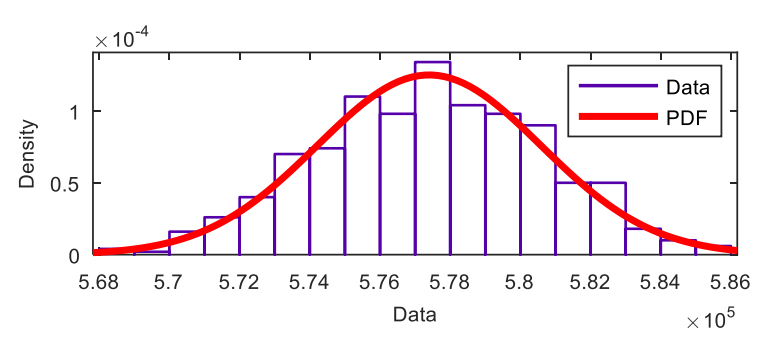

Figure 9. Cost objective function without DR

Table 4. Gaussian parameters for the cost objective function with DR and without DR

\begin{tabular}{cccc}
\hline Parameter & $\begin{array}{c}\text { Estimate } \\
\text { without DR }\end{array}$ & $\begin{array}{c}\text { Estimate } \\
\text { with DR }\end{array}$ & Difference \\
\hline Mean & $\$ 577,400$ & $\$ 569,120$ & $\$ 8,271$ \\
standard deviation & $\$ 3,190$ & $\$ 3,360$ & - \\
\hline
\end{tabular}

In order to quantify DR benefits, others cost functions are set up to provide insight about the dispatching cost. Table 5 provides six cost functions for demand response. This cost functions represents the willing of each load to provide DR. The plot for those cost functions are shown in Figure 10.

Table 5. Incentive based demand response

\begin{tabular}{cc}
\hline Ref. & Cost Function $[\$]$ \\
\hline $\mathrm{A}$ & $C_{A}=0.000128 P^{2}+1.4 P+439$ \\
$\mathrm{~B}$ & $C_{B}=0.00248 P^{2}+2.4 P+449$ \\
$\mathrm{C}$ & $C_{C}=0.00128 P^{2}+6.4 P+459$ \\
$\mathrm{D}$ & $C_{D}=0.00528 P^{2}+6.4 P+469$ \\
$\mathrm{E}$ & $C_{E}=0.00928 P^{2}+6.4 P+479$ \\
$\mathrm{~F}$ & $C_{F}=0.015221 P^{2}+6.4 P+489$ \\
\hline
\end{tabular}

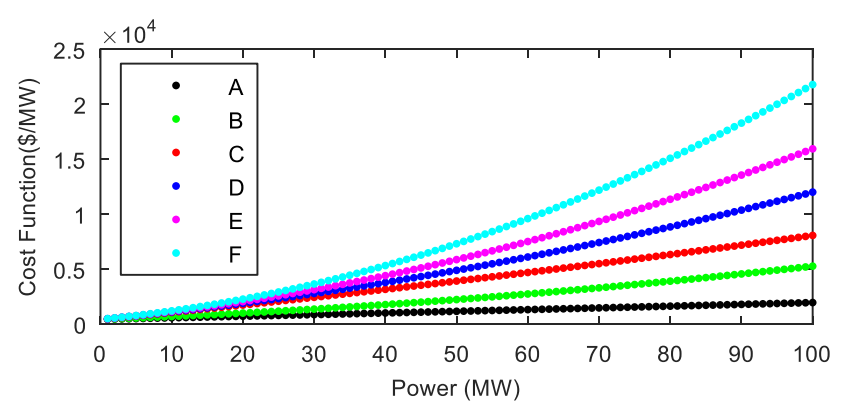

Figure 10. Cost functions for demand response

The power generation cost varies according to different incentives for DR as shown in Figure 11. For instance, the case F, in Figure 10, shows a cost function with high bid for DR. The case A corresponeds to a situation with a lower cost function, however, the dispatching is not the lower. For the cases evaluated, the optimal solution corresponds to the case $\mathrm{C}$, the cost function $\mathrm{C}$ is between the cases $\mathrm{A}$ and $\mathrm{F}$. 


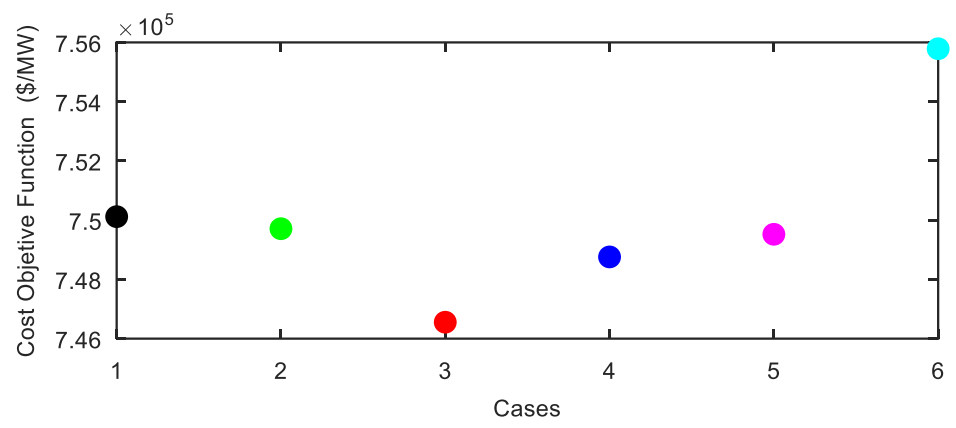

Figure 11. Cost objective function for different incentives to DR

\section{CONCLUSION}

In this paper, presented an integrated OPF model that explicitly includes wind power generation and demand response resources for day-ahead dispatching in constrained electricity markets. Demand response is integrated into the model as flexible loads with willing to bid day-ahead. Observed that considerable savings in power generation cost could be achieved if the demand participates in the markets. The numerical results show that the wind power uncertainty can be captured using trajectories from a Monte-Carlo simulation.

\section{ACKNOWLEDGEMENTS}

The authors acknowledge the support of the Universidad Autónoma de Occidente in Colombia.

\section{REFERENCES}

[1] J. M. Morales, et al., "Short-term trading for a wind power producer," IEEE Transactions on Power Systems, vol/issue: 25(1), pp. 554-564, 2010.

[2] S. S. Sakthi, et al., "Wind Integrated Thermal Unit Commitment Solution using Grey Wolf Optimizer," International Journal of Electrical and Computer Engineering (IJECE), vol/issue: 7(5), pp. 2309-2320, 2017.

[3] I. M. Wartana, et al., "Optimal Integration of the Renewable Energy to the Grid by Considering Small Signal Stability Constraint," International Journal of Electrical and Computer Engineering (IJECE), vol/issue: 7(5), pp. 2329-2337, 2017.

[4] C. L. Su and D. Kirschen, "Quantifying the effect of demand response on electricity markets," IEEE Transactions on Power Systems, vol/issue: 24(3), pp. 1199-1207, 2009.

[5] R. A. Jabr and B. C. Pal, "Intermittent wind generation in optimal power flow dispatching," IET Gener. Transm. Distrib, vol/issue: 3(1), pp. 66-74, 2009.

[6] H. Zhang and P. Li, "Probabilistic analysis for optimal power flow under uncertainty," IET Gener. Transm. Distrib, vol/issue: 4(5), pp. 553-561, 2010.

[7] R. Entriken, et al., "Stochastic optimal power flow in systems with wind power," Proc. IEEE Power Energy Soc. Gen. Meeting, San Diego, CA, USA, pp. 1-5, 2011.

[8] C. S. Saunders, "Point estimate method addressing correlated wind power for probabilistic optimal power flow," IEEE Trans. Power Syst., vol/issue: 29(3), pp. 1045-1054, 2014.

[9] A. Papavasiliou and S. S. Oren, "Multiarea stochastic unit commitment for high wind penetration in a transmission constrained network," Oper. Res., vol/issue: 61(3), pp. 578-592, 2013.

[10] F. Bouffard and F. D. Galiana, "Stochastic security for operations planning with significant wind power generation," IEEE Trans. Power Syst., vol/issue: 23(2), pp. 306-316, 2008.

[11] J. M. Morales, et al. "Economic valuation of reserves in power systems with high penetration of wind power," IEEE Trans. Power Syst., vol/issue: 24(2), pp. 900-910, 2009.

[12] A. Papavasiliou, et al., "Reserve requirements for wind power integration: A scenario-based stochastic programming framework," IEEE Trans. Power Syst., vol/issue: 26(4), pp. 2197-2206, 2011.

[13] B. Banhtasit and C. S. Dechanupaprittha, "Optimal Generation Scheduling of Power System for Maximum Renewable Energy Harvesting and Power Losses Minimization," International Journal of Electrical and Computer Engineering (IJECE), vol/issue: 8(4), pp. 1954-1966, 2018.

[14] S. Kim and S. R. Salkut, "Optimal power flow based congestion management using enhanced genetic algorithms," International Journal of Electrical and Computer Engineering (IJECE), vol/issue: 9(2), pp. 875-883, 2019.

[15] M. Kefayati and R. Baldick, "Harnessing demand flexibility to match renewable production using localized policies," Proc. 50th Annu. Allerton Conf. Commun. Control Comput. (Allerton), Monticello, IL, USA, pp. 1105$1109,2012$.

[16] U.S. Energy Information Administration (EIA), "Estimated U.S. Residential Electricty Consumption by End-Use," 2010. Available: http://www.eia.gov/tools/faqs/faq.cfm?id=96\&t=3 
[17] M. Arroyo and A. J. Conejo, "Multiperiod auction for a pool-based electricity market," IEEE Trans. Power Syst., vol. 17, pp. 1225-1231, 2002.

[18] J. Wang, et al., "Demand-side reserve offers in joint energy/reserve electricity markets," IEEE Trans. Power Syst., vol. 18, pp. 1300-1306, 2003.

[19] A. Borghetti, et al., "Auctions with explicit demand side bidding in competitive electricity markets," The Next Generation of Electric Power Unit Commitment Models. Norwell, MA: Kluwer, pp. 53-74, 2001.

[20] O. Ma, et al., "Demand Response for Ancillary Services," IEEE Trans. Smart Grid, vol. 4, pp. 1988-1995, 2013.

[21] U. Helman, et al., "Operational requirements and generation fleet capability at 20\% RPS," CAISO, 2010. Available: http://www.uwig.org/

[22] G. Lazaros, et al., "The role of aggregators in smart grid demand response markets," IEEE Journal on Selected Areas in Communications, vol/issue: 31(7), pp. 1247-1257, 2013.

[23] The GUROBI Manual, 2017. Available: https://www.gurobi.com/documentation/7.5/refman/index.html.

[24] Matpower Optimal Scheduling Tool (MOST) package, 2017. Available: http://www.pserc.cornell.edu/ matpower/manual.pdf

[25] R. Z. Miñano, et al., "An OPF Methodology to Ensure Small-Signal Stability," IEEE Trans. Power System, vol/issue: 26(3), 2011.

[26] T. Dai, et al., "Real-time Optimal Participation of Wind Power in an Electricity Market," IEEE Innovative Smart Grid Technologies Conf., Tianjin, China, 2012.

[27] S. Jang, et al., "A new network partition method using the sensitive of marginal cost under network congestion," IEEE Power Engineering Society Summer Meeting, 2001. 